\title{
Caracterização Mecânica da Região Soldada de Tailor Welded Blanks (TWB) a Partir do Perfil de Microdureza
}

\author{
Guilherme Souza Assunção ${ }^{1}$ (D) , Etiene Pereira de Andrade ${ }^{1}$ (D), Wellington Augusto dos Santos ${ }^{2}$, Ivanilza Felizardo ${ }^{3}$, \\ Alexandre Queiroz Bracarense ${ }^{1}$ \\ ${ }^{1}$ Universidade Federal de Minas Gerais - UFMG, Belo Horizonte, MG, Brasil. \\ ${ }^{2}$ FIAT Chrysler Automóveis do Brasil LTDA, Betim, MG, Brasil. \\ ${ }^{3}$ Centro Federal de Educação Tecnológica de Minas Gerais - CEFET-MG, Belo Horizonte, MG, Brasil.
}

Como citar: Assunção GS, Andrade EP, Santos WA, Felizardo I, Bracarense AQ. Caracterização mecânica da região soldada de Tailor Welded Blanks (TWB) a partir do perfil de microdureza. Soldagem \& Inspeção. 2019;24:e2432. https://doi.org/10.1590/0104-9224/SI24.32

\begin{abstract}
Resumo: No Tailor Welded Blank (TWB) são soldadas chapas de especificações distintas, antes da conformação. Sua caracterização, na região soldada, por métodos convencionais de tração, pode ser dificultada pelo estreito comprimento da solda. Novas metodologias e alternativas são analisadas. Este trabalho visou caracterizar mecanicamente a região soldada de um painel interno de porta veicular, fabricado por TWBs. Realizou-se a caracterização mecânica indireta da região soldada a partir de ensaio de microdureza - normas ASTM-E140 e ISO 18265-2013 - e equação de conversão direta de dureza em limite de resistência à tração (LRT). Os resultados obtidos foram correlacionados com ensaio de tração dos materiais de base, evidenciando qual a melhor conversão. A norma ISO 18265-2013 foi a que mais se aproximou do ensaio direto, com desvio máximo de $30 \%$ e mínimo de $9 \%$, sendo o desvio mais acentuado conforme a resistência mecânica do material aumenta. Nos outros métodos, o desvio máximo pode chegar a $40 \%$, sendo menor (11\% a $18 \%$, para equação) quando o material apresenta menor LRT. Apesar do desvio observado, a técnica utilizada mostrou-se útil para predizer os estados de deformações e a extensão da zona fundida (ZF) e da zona termicamente afetada (ZTA) de um TWB.
\end{abstract}

Palavras-chave: Conformação; Soldagem a laser; Tailor Welded Blank; Indústria automobilística.

\section{Mechanical Characterization of the Welded Region of Tailor Welded Blanks (TWB) from the Microhardness Profile}

\begin{abstract}
Tailor Welded Blank (TWB) are plates of different specifications welded before forming. The narrow weld length may hamper its characterization in the welded region by conventional tensile methods. New methodologies and alternatives are analyzed. This work aimed to mechanically characterize the welded region of an internal vehicle door panel, manufactured by TWBs. Indirect mechanical characterization of the welded region was performed from micro hardness test - ASTM-E140 and ISO 18265-2013 - and the direct conversion of hardness to ultimate tensile strength (UTS) equation. The results were correlated with tensile test of the base materials, showing the best conversion. ISO 18265-2013 was the one that came closest to the direct test, with a maximum deviation of $30 \%$ and a minimum of $9 \%$, with the most pronounced deviation as the mechanical strength of the material increases. In other methods, the maximum deviation can reach $40 \%$, being smaller ( $11 \%$ to $18 \%$, for equation) when the material has lower UTS. Despite the observed deviation, the technique used proved to be useful for predicting the deformation states and the extent of the weld zone (WZ) and the heat affected zone (HAZ) of a TWB.
\end{abstract}

Key-words: Forming; Laser welding; Tailor Welded Blank; Auto industry.

\section{Introdução}

A indústria automobilística tende a se renovar a cada dia em busca de desenvolvimento tecnológico, acompanhando as tendências de mercado e normas de exigência mundial. Destaca-se dentre as características desejadas aos novos veículos, baixo consumo de combustível, redução da emissão de poluentes e aumento da segurança dos ocupantes. Tem-se então automóveis mais eficientes, sustentáveis e seguros [1].

Nessa vertente, o processo de fabricação de componentes da carroceria de um veículo por Tailor Welded Blanks (TWB) confere redução de peso e boa resistência mecânica a tração ao blank por meio da união de chapas com diferentes características (material, espessura, revestimento e propriedades físicas/químicas) [2-5]. Esse processo de união é feito por 
processos de soldagem não convencionais, normalmente a laser, antes do processo de conformação, para obtenção do produto final [6].

Geralmente o blank é composto por duas chapas, na qual o material menos espesso (via de regra, de menor resistência mecânica), poderá se deformar mais que o de maior espessura e resistência mecânica, nas regiões de conformação severa. Observa-se então um fenômeno conhecido como movimentação da linha de solda, podendo levar essa região à fratura e a falhas, geralmente do tipo 1 (iniciada na linha de solda: a deformação e a tensão principais são paralelas à linha de solda, que se rompe devido à sua menor ductilidade) e do tipo 2 (iniciada na região de menor resistência mecânica: o esforço principal se concentra perpendicularmente à linha de solda) [7,8]. Deve-se trabalhar, portanto, com ferramentais que possuam sistema de compensação, o qual acomoda as diferentes espessuras das chapas, permitindo o fluxo uniforme e controlado do material [9,10].

Soldabilidade e conformabilidade são os dois principais conceitos abordados na utilização de TWBs [11]. A soldabilidade, em TWBs é afetada principalmente pela composição química dos materiais de base, podendo estar atrelada também à espessura das chapas, e, sobretudo à vazão do gás de proteção [12]. Esse conjunto de variáveis é utilizado para determinar o processo de soldagem mais adequado [13]. Via de regra, trabalha-se com feixes de laser [14]. A razão de espessura (razão entre a maior e menor espessura das chapas que compõe o blank) e o posicionamento do cordão de solda são frequentemente avaliados, averiguando sua influência na conformação dos componentes. É comum se trabalhar com curvas limites de conformabilidade, ensaios de tração e simulações [15].

Na maioria dos ensaios, uma vez qualificada a solda, evidencia-se que as potenciais falhas do blank fabricado por TWB ocorrem em torno da região soldada, devido à concentração de tensão da região. Isso ocorre em virtude da diferença entre as propriedades mecânicas dos materiais de base (MBs), da zona fundida (ZF) e da zona termicamente afetada (ZTA) [10].

No ensaio de tração, a contribuição da linha de solda no corpo de prova tende a ser reduzida, uma vez que o processo de soldagem a laser produz soldas muito estreitas. Tem-se duas formas de se extrair os corpos de prova: com a linha de solda posicionada paralela ou perpendicular à direção de tração. Na primeira opção, tem-se a solda com cerca de $1 \mathrm{~mm}$ para uma alma do corpo de prova com $6 \mathrm{~mm}$. No segundo caso, a amostra se romperá no material de menor espessura e menor resistência mecânica. Caso a ruptura ocorra na solda, há fortes indícios de defeitos de fabricação [15-19].

O presente trabalho tem o objetivo de caracterizar mecanicamente a região soldada de um blank fabricado por TWB. Devido a restrições e dificuldades encontradas para caracterizar a região soldada a partir de ensaio de tração com corpos de prova de tamanho padrão, baseado na norma ASTM-E8 [20], optou-se por fazer a caracterização mecânica indireta, por meio de ensaios de microdureza, trabalhando-se com a norma ASTM-E3 [21], para preparação metalográficas e ASTM E-384 [22] para microdureza propriamente dita.

Optou-se então, neste trabalho, pela análise indireta por meio de ensaios de microdureza, normas e equações de conversão de dureza em limite de resistência a tração. Para melhor correlação entre os dados obtidos pela caracterização indireta, foram feitos ensaios de tração nos metais de base.

\section{Metodologia}

Foram realizados ensaios de tração uniaxial, microdureza, metalografia e uso de normas para obter os resultados deste estudo. Utilizou-se dois blanks fornecidos pela FCA Brasil para verificar a influência do processo de conformação na região soldada e na comparação dos dados de resistência mecânica a tração das chapas obtidas pelos ensaios direto e indireto (ensaio de tração e microdureza, respectivamente). Para tal, foram utilizados um blank sem conformação e outro com todas as etapas de conformação necessarias para utilização da chapa como um painel interno de porta de um automóvel.

Nas Tabela 1 e 2 são apresentadas as propriedades mecânicas por tração e composições químicas dos materiais fornescidos pela CSN (Companhia Siderúgica Nacional) que foram utilizados na confecção do blank. Essas chapas foram soldadas por laser de $\mathrm{CO}_{2}$, com potência nominal de $8 \mathrm{~kW}$. Foi utilizada potência média de $65 \%$, gás de proteção de hélio puro com vazão de $10,5 \mathrm{~L} / \mathrm{min}$ e velocidade de soldagem de $6,5 \mathrm{~m} / \mathrm{min}$.

Tabela 1. Materiais utilizado na confecção do TWB e suas propriedades mecânicas por tração.

\begin{tabular}{|c|c|c|c|}
\hline Material & Espessura $[\mathrm{mm}]$ & $\begin{array}{l}\text { Limite de escoamento (LE) } \\
{[\mathrm{MPa}]}\end{array}$ & Limite de Resistência a Tração (LRT) [MPa] \\
\hline Chapa 1: Aço CSN FEP05 & 0,7 & 157 & 284 \\
\hline Chapa 2: Aço CSN FEE210 & 1,2 & 217 & 346 \\
\hline
\end{tabular}

Tabela 2. Composição química dos aços utilizados na confecção do TWB.

\begin{tabular}{ccccccccc}
\hline Elemento (\%) & $\mathbf{C}$ & $\mathbf{M n}$ & $\mathbf{P}$ & $\mathbf{S}$ & $\mathbf{S i}$ & $\mathbf{N}$ & $\mathbf{N b}$ & $\mathbf{T i}$ \\
AÇO CSN IF FEP 05 & 0,0018 & 0,1350 & 0,0120 & 0,0100 & 0,0050 & 0,00420 & 0,0030 & 0,0570 \\
AÇO CSN IF FEE 210 & 0,0021 & 0,4320 & 0,0360 & 0,0120 & 0,0050 & 0,00360 & 0,0024 & 0,0260 \\
\hline
\end{tabular}


O blank obtido após a etapa de soldagem a laser das duas chapas base (Aço CSN FEP05 e Aço CSN FEE210), está apresentado na Figura 1.

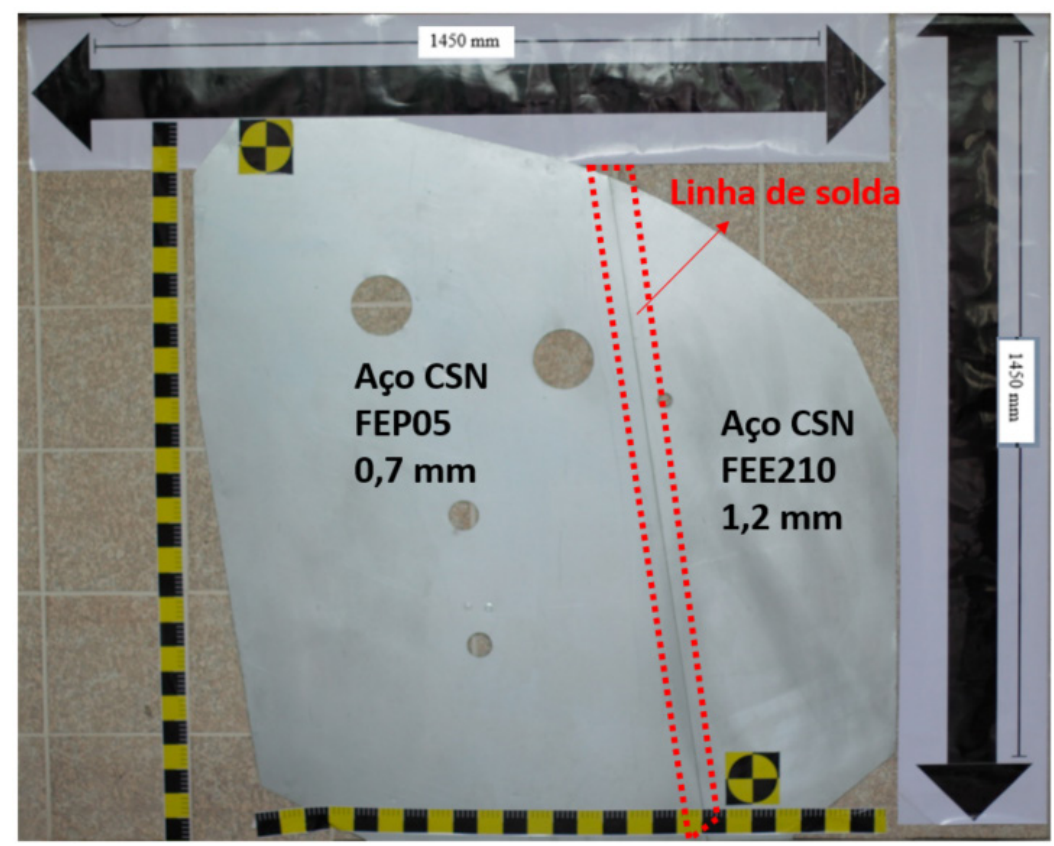

Figura 1. Blank fabricado por TWB sem estampagem.

Após as etapas de estampagem e corte da segunda chapa plana, o blank, se torna um painel interno de porta de um veículo, apresentado na Figura 2A. Devido ao tamanho do componente, foi realizado corte conforme a linha vermelha tracejada na Figura 2A, região na qual compreende a linha de solda e regiões de grande solicitação da conformação (região de fixação do auto falante na porta do veículo). Dessa forma, a peça de analise é apresentada na Figura 2B e C.

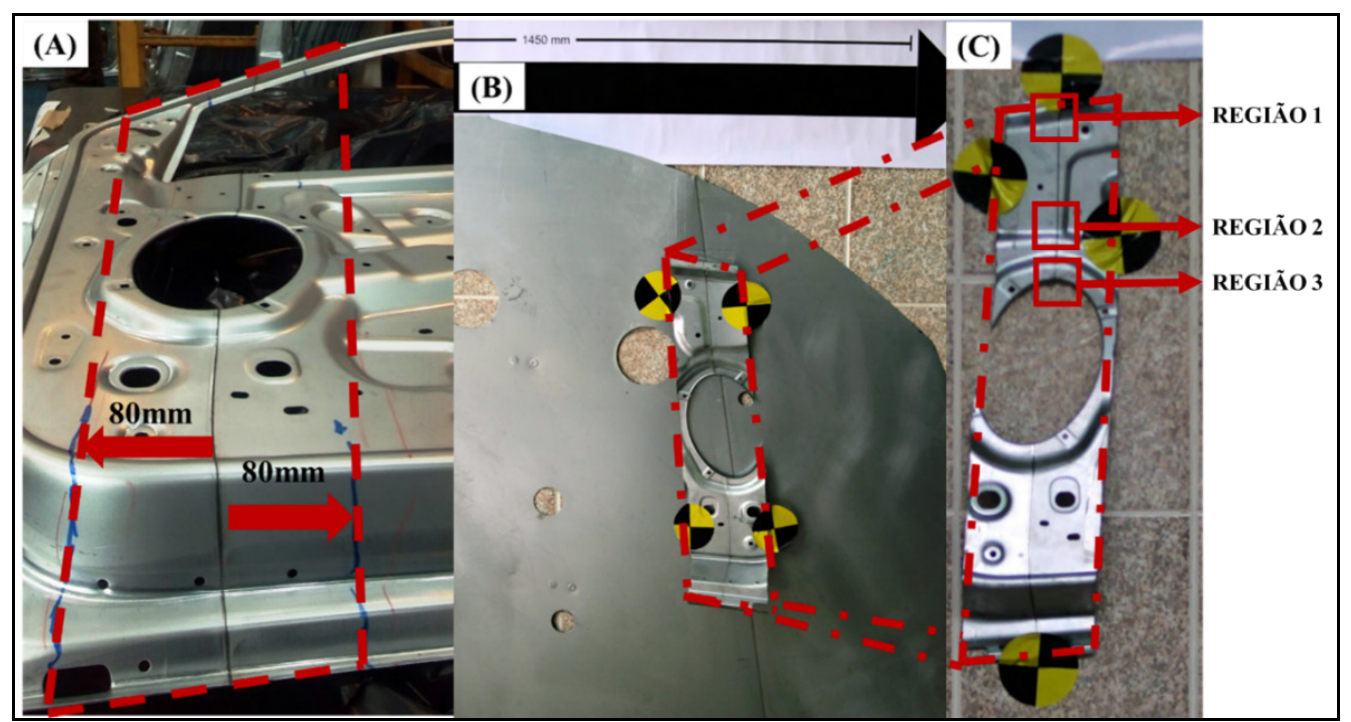

Figura 2. (A) Blank fabricado por TWB submetido a estampagem e recortes; (B) Blanck recordado na região de maior solicitação (região de fixação do auto falante do veículo; (C) Regiões de análise do blank estampado.

Para verificação da resistência mecânica a tração do blank não conformado foram confeccionados corpos de prova para ensaio de tração conforme a Figura 3B, com base na norma ASTM E8 [20]. O ensaio foi realizado com célula de carga de 10kN, extensômetro óptico e velocidade de deformação de $0,001 \mathrm{~s}^{-1}$, numa máquina de ensaios universal Instron ${ }^{\circledR} 5582$.

Os corpos de prova do ensaio de tração foram dispostos conforme a Figura 3A. Foram posicionados três corpos de prova para o material FEP05 e três para o material FEE210, conforme a Figura 3E. Outras três, com cordão de solda disposto no meio da amostra, perpendicular à direção de tração, para verificar a integridade da linha de solda, como apresentado na Figura 3C. 


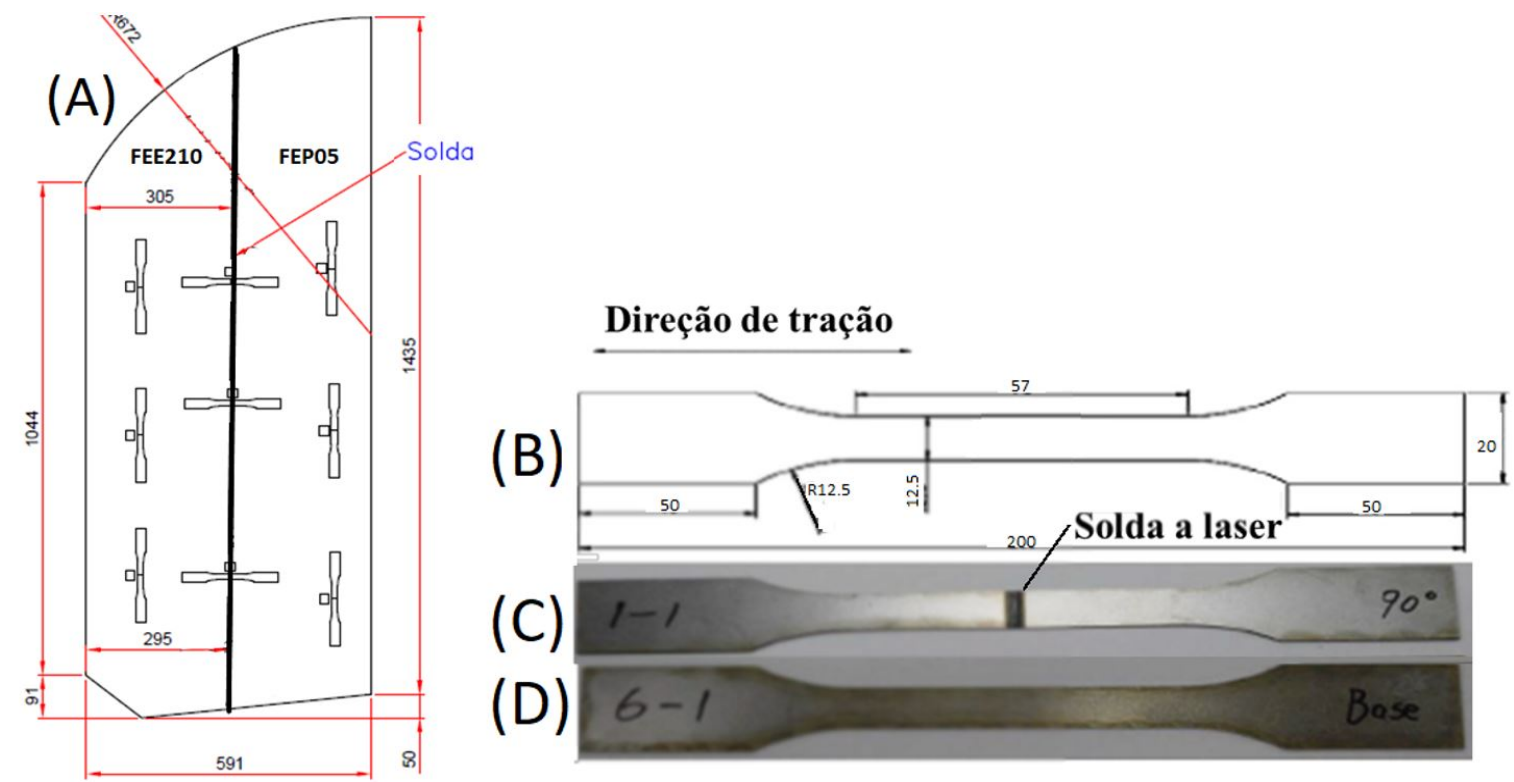

Figura 3. (A) Regiões de retirada dos corpos de prova; (B) Tamanho dos corpos de prova; (C) Corpo de prova com a solda posicionada a $90^{\circ}$ em relação a direção de tração; (D) Corpo de prova do material de base, sem solda.

Após a verificação da resistência mecânica do blank, a partir do ensaio de tração, foram confeccionados corpos de prova para a realização do teste de microdureza e ensaio de metalografia. Retirou-se três amostras do blank submetido à conformação, e uma da chapa não conformada, resultando em quatro amostras selecionadas: não conformada (N.C) e conformadas (C1, C2 e C3).

Os corpos de prova foram escolhidos conforme o nível de deformação durante a estampagem. Na chapa estampada foram selecionadas três regiões distintas como apresentado na Figura 2 C, são elas: C1, região sujeita à compressão do prensa chapas, C2, área plana sem conformação visível e C3, região na qual vai fixado o autofalante da porta do veículo e a conformação é evidenciada a olho nu. A amostra não conformada foi retirada aleatoriamente, ao longo da linha de solda apresentada na Figura 3A.

Essas amostras foram cortadas mecanicamente, com auxílio de guilhotina, e em seguida, foram embutidas em resina epóxi para a realização do lixamento. Utilizou-se lixas no sentido crescente de granulometria: \#80, \#220, \#320, \#400, \#600, \#1200 e \#1500. O polimento subsequente foi feito com pasta de alumina $\left(\mathrm{Al}_{2} \mathrm{O}_{3}\right)$ em suspensão de $1 \mu \mathrm{m}$, utilizado pano de polimento metalográfico aveludado específico para alumina [21].

O ataque químico das amostras foi feito com Nital a 5\%, de modo direto, sem imersão, utilizando a técnica de esfregaço, sendo 10 esfegraços em cada sentido.

O ensaio de dureza foi realizado segundo norma ASTM E384 [22]. Destaca-se como principais parâmetros de configuração do ensaio a carga aplicada e seu tempo de permanência. Utilizou-se a carga de 300gf aplicada por 15s, além de uma distância de $300 \mu \mathrm{m}$ entre as endentações na região do material de base e de $200 \mu \mathrm{m}$ nas regiões da ZTA e ZF.

Após o ensaio de microdureza, as amostras foram levadas ao microscópio acoplado à câmera para coleta de imagens e análises metalográficas. Buscou-se distinguir os materiais de base (MB), a zona termicamente afetada (ZTA) e a zona fundida (ZF). Além disso, foram visualizados os efeitos da conformação e comparadas as microestruturas das amostras conformadas com a não conformada.

Os resultados dos ensaios de microdureza foram convertidos em tensão (MPa), tornando viável a comparação das propriedades mecânicas dos materiais de base com a região soldada. Para isso, utilizou-se dois métodos de conversão de dureza (HV) em limite de resistência à tração (LRT): as normas ASTM-E140 [23] e ISO 18265-2013 [24].

A norma ASTM-E140 [23], Tabela 2, transforma a dureza Vickers (HV) em Brinell (HB) para aços não austeníticos, e com isso é possível utilizar a Equação 1 para determinar a tensão limite de resistência à tração (LRT) do material analisado [25].

$\operatorname{LRT}(M P a)=3,45 x H B$

A norma ISO 18265-2013, Anexo A, Tabela A.1, para aços não ligados, de baixa liga e aços fundidos, possui duas formas de transformação de dureza em LRT. A primeira forma é como a utilizada na norma ASTM-E140, na qual tranforma a dureza Vickers (HV) em Brinell (HB) e posteriormente usa-se a Equação 1 para obter o LRT. Já a segunda forma, apresenta conversão direta de dureza Vickers (HV) em LRT, ou seja, através de um ensaio experimental prévio, tem-se a correlação entre a tensão limite de resisntecia a tração e a dureza.

Dessa forma, foi possível correlacionar as três conversões, uma para norma ASTM-E140 e duas para a norma ISSO 18265-2013, para verificar qual se aproxima mais da realidade (ensaio de tração). 


\section{Resultados e Discussão}

A partir do ensaio de tração obteve-se os dados da tensão limite de resistência à tração (LRT) dos materiais de base e da amostra com a solda perpendicular à direção de tração, sendo os resultados apresentados na Tabela 3.

Tabela 3. Ensaio de tração uniaxial.

\begin{tabular}{|c|c|c|c|}
\hline \multirow[t]{2}{*}{ Testes } & Chapa $1(0,7 \mathrm{~mm})$ - FEP05 & Chapa $2(1,2 \mathrm{~mm})$ - FEE210 & $\begin{array}{l}\text { Amostra com linha de solda } \\
\text { perpendicular a direção de tração }\end{array}$ \\
\hline & \multicolumn{3}{|c|}{ Limite de resistência a tração ( $\mathrm{MPa}$ ) } \\
\hline 1 & 287 & 347 & 302 \\
\hline 2 & 294 & 353 & 299 \\
\hline 3 & 285 & 352 & 299 \\
\hline MÉDIA & 289 & 351 & 300 \\
\hline Desvio padrão & 3,86 & 2,62 & 1,41 \\
\hline
\end{tabular}

Os dados obtidos a partir do ensaio de tração para os materiais de base são próximos aos declarados pelo fabricante (Tabela 1). Houve variação de $4 \%$ para o FEP05 e de $2 \%$ para o FEE210, resultado satisfatório para ensaios feitos em equipamentos e locais distintos.

Em relação à amostra com a linha de solda perpendicular à direção de tração, verifica-se LRT bem próximo ao material de menor resistência. A Regras das Misturas (Rule Of Mixtures - ROM) juntamente com o principio de Saint Venant justficam este fato.

A ROM, segundo a Equação 2, mostra que a resistência tração da solda é intermédiaria a resistência a tração dos materiais de base [17]. O principio de Saint Venant, diz que um material isótropo, contínuo e homogênio deveria romper no meio do corpo de prova, porém o corpo de prova possui materiais dissimilares, anisotrópicos e descontínuos, que leva a ruptura dos corpos de prova em lugares aleatórios no material de menor espessura $[25,26]$.

$\sigma_{w}=\frac{P-\left(K_{1} \cdot \varepsilon_{1}^{n_{l}}\right) \cdot A_{1}-\left(K_{2} \cdot \varepsilon_{2}^{n_{2}}\right) \cdot A_{2}}{A_{w}}$

na qual: $\sigma_{\mathrm{w}}=$ Tensão limite de resistência da solda [MPa]; $\mathrm{P}=$ Carga total suportada pelo corpo de prova [N]; $\mathrm{K}_{1}=$ Coeficiente de resistência mecânica do material 1 do TWB [MPa]; $\varepsilon_{1}=$ Deformação verdadeira do material 1 do corpo de prova do TWB; $\mathrm{A}_{1}=$ Área da seção transversal do material 1 do corpo de prova do TWB [mm²]; $K_{2}=$ Coeficiente de resistência mecânica do material 2 do TWB [MPa]; $\varepsilon_{2}=$ Deformação verdadeira do material 2 do TWB; $A_{2}=$ Área da seção transversal do material 2 do corpo de prova do TWB [MPa]; $A_{w}=$ Área da seção transversal da solda do corpo de prova do TWB [mm²].

As amostras se romperam sempre no material FEP05, conforme a Figura 4, pois é o material de menor espessura e resistência mecânica a tração. Isso implica em uma solda com boa qualidade e de maior resistência mecânica que os materiais de base como previsto pela ROM [17,18] e por uma das principais referências indicadas para TWB [27].

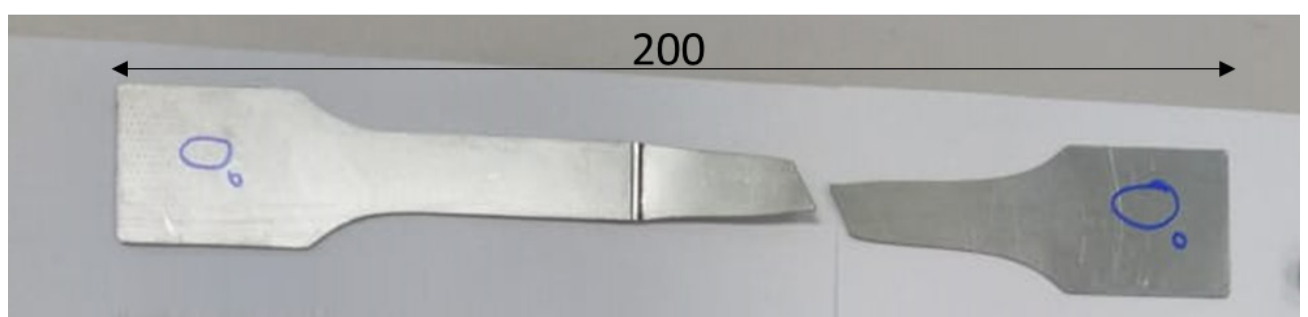

Figura 4. Amostra rompida com a linha de solda perpendicular a direção de tração.

Apesar disso, não houve um padrão da fratura, a qual ocorreu de forma randômica, em locais variados do corpo de prova do material de menor espessura, conforme constatado também em trabalhos anteriores [4,5]. Ainda assim, mesmo sem repetitividade dessa característica, pode-se assegurar boa qualidade da solda pois o material não rompeu na área soldada [10].

Os ensaios de microdureza e micrografia estão apresentados na Figuras 5 a 9 . 0 gráfico de microdureza apresenta a média dos três ensaios para cada uma das quatro amostras (N.C, C1, C2 e C3) analisadas. As endentações oriundas desses ensaios são evidentes nas metalografias das Figuras 6 a 9. Adotou-se o seguinte padrão: à esquerda tem-se o material de menor resistência mecânica e consequentemente menor dureza; à direita, o material mais resistente, espesso e duro. 


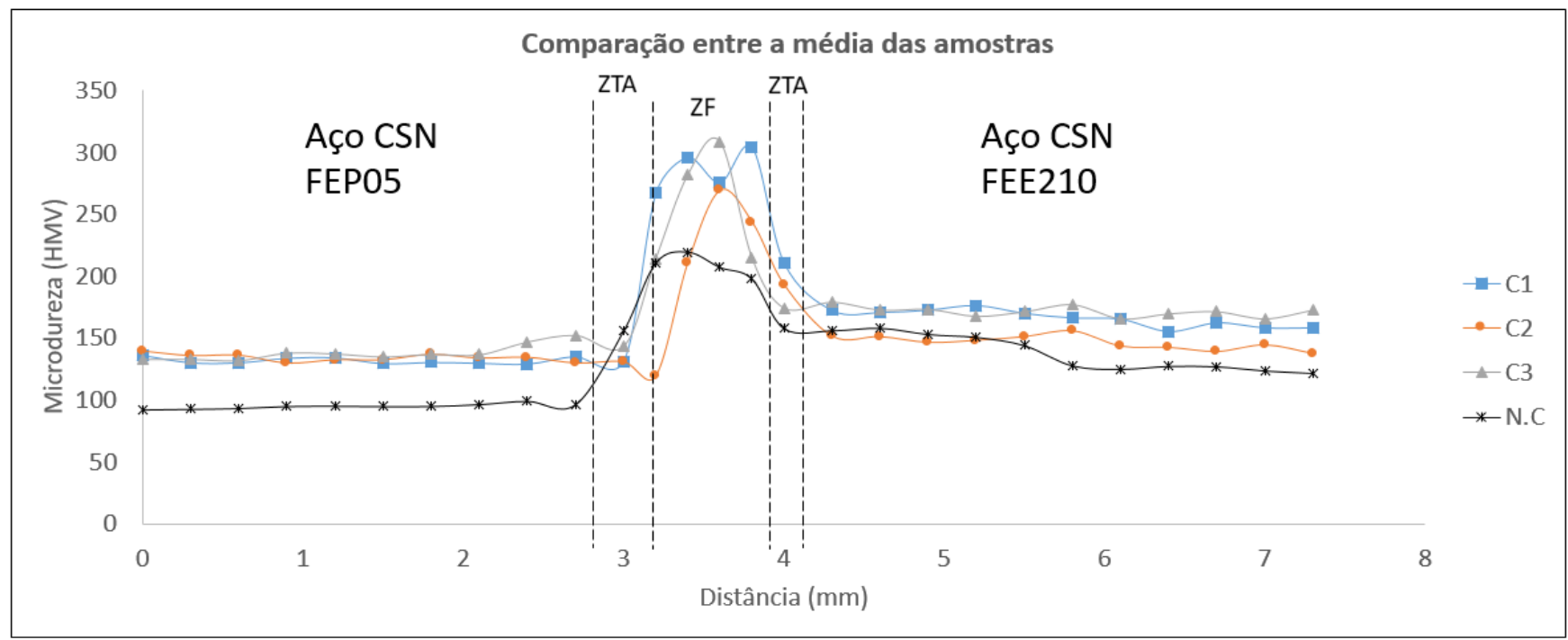

Figura 5. Perfil de microdureza das amostras N.C, C1, C2 e C3. ZTA (Zona termicamente afetada) e ZF (Zona Fundida).

Observa-se que a amostra não conformada (N.C) apresenta dureza inferior às amostras conformadas (C1, C2 e C3), conforme esperado. As amostras conformadas foram submetidas à maior deformação do material, o que leva ao encruamento, com consequente elevação da resistência mecânica, refletindo em maior dureza. Esse fato é mais evidente no material de menor resistência mecânica, o que leva à inferência de que o material de maior espessura se sujeita a menor fluxo e deformação durante a estampagem.

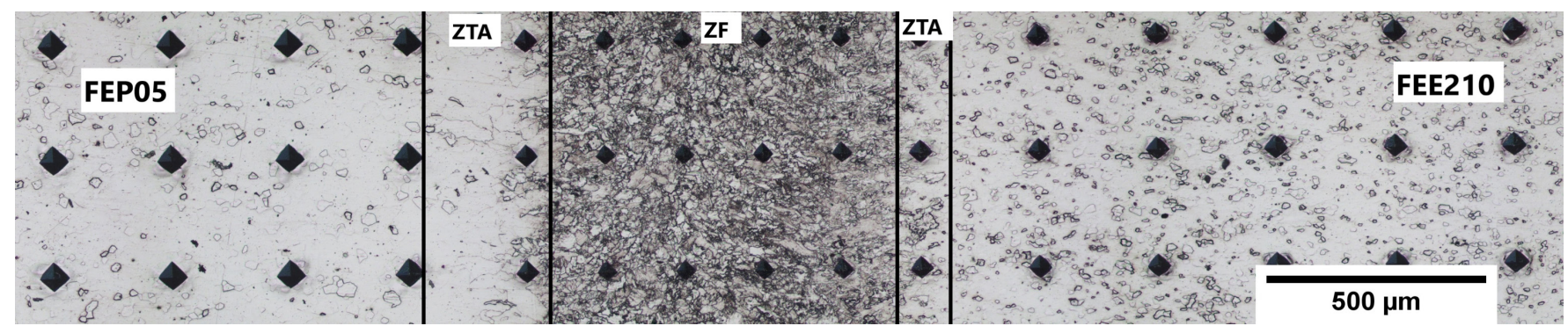

Figura 6. Ensaio de metalografia das amostras N.C. ZTA (Zona termicamente afetada) e ZF (Zona Fundida).

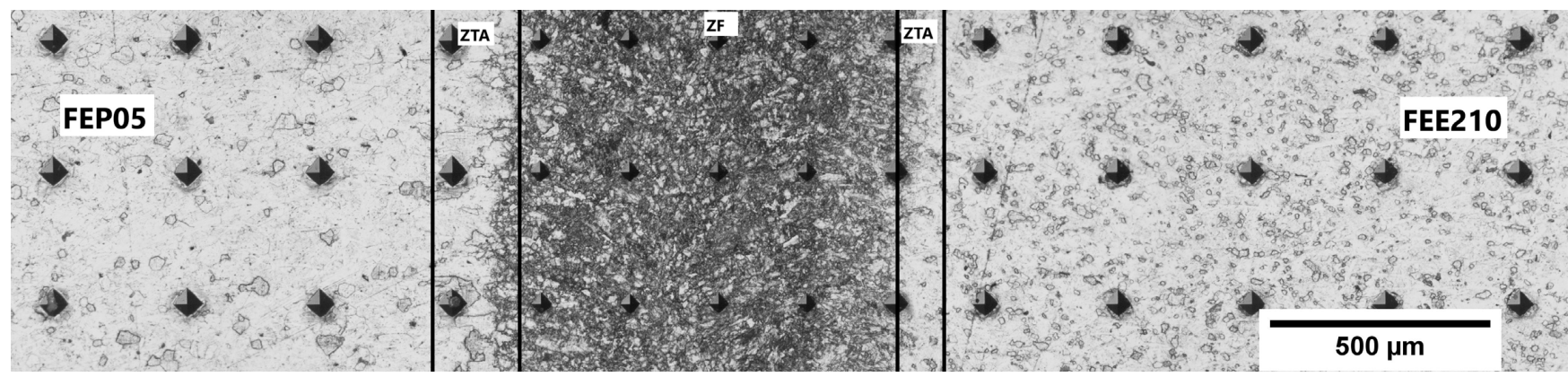

Figura 7. Ensaio de metalografia das amostras C1. ZTA (Zona termicamente afetada) e ZF (Zona Fundida). 


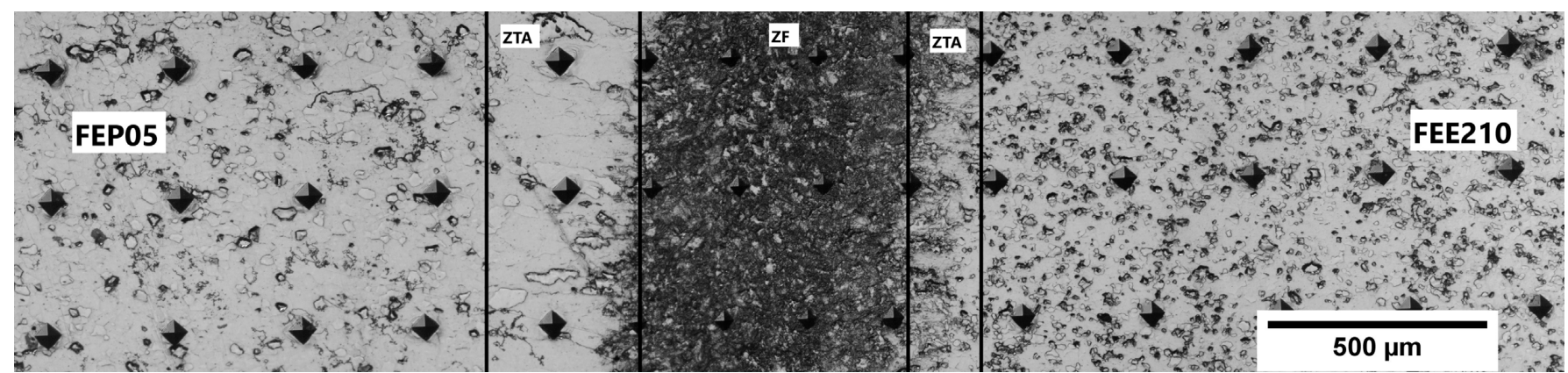

Figura 8. Ensaio de metalografia da amostra C2. ZTA (Zona termicamente afetada) e ZF (Zona Fundida).

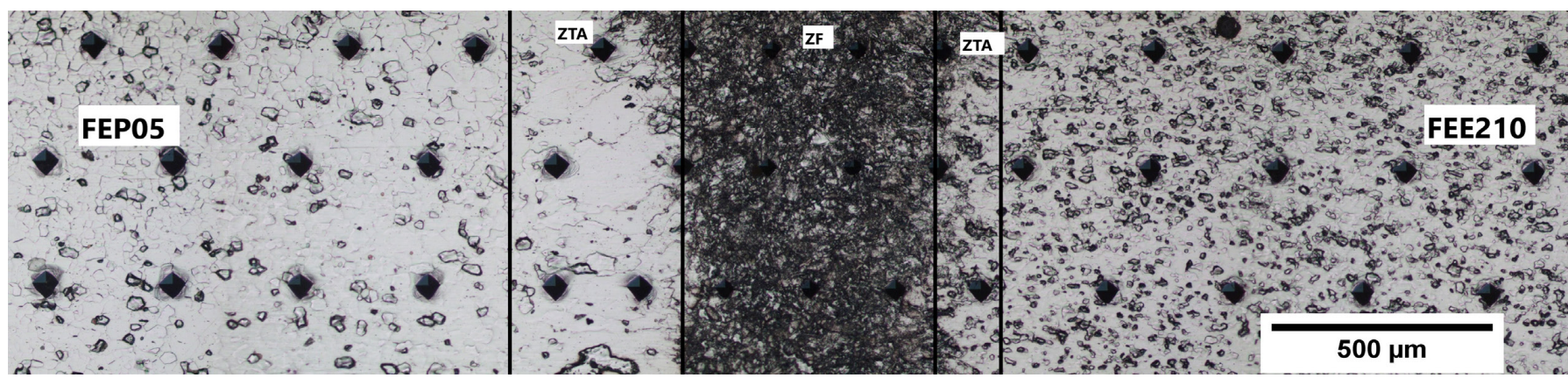

Figura 9. Ensaio de metalografia da amostra C3. ZTA (Zona termicamente afetada) e ZF (Zona Fundida).

Observa-se, em ambos os ensaios (micrografia e microdureza), que a ZTA do material de menor espessura e resistência mecânica (FEP05) é maior que o mais resistente e espesso (FEE210). A ZTA extensa causa "amaciamento" entre o material FEP05 e a ZF, o que pode causar uma perda de resistência local e tornar-se um ponto frágil durante o processo de conformação posterior a fabricação do TWB $[19,28,29]$. Entretanto, conforme verificou-se no ensaio de tração, essa perda de resistência local não causou fraturas na região soldada nos testes efetuados [20,30].

Os limites de resistência a tração (LRT) obtidos a partir das normas ASTM E140 [23] e ISO 18265-2013 [24], estão apresentados na Tabela 4. Os dados de microdureza são resultados da média de cada uma das regiões análisadas. Foram feitas as conversões para os materiais de base (FEP05 e FEE210), e também para a região soldada na qual inclui a zona termicamente afetada e zona fundida.

Os limites de resistência a tração analisados são conforme a norma ASTM E140 [23] e ISO 18265-2013 [24]. A norma ISSO 18265-2013 possui duas formas de conversão, a primeira, igualmente a norma ASTM E140, leva em consideração a conversão de dureza Vickers (HV) em Brinell (HB) e depois utilizada-se a Equação 1 para obter o LRT. A segunda conversão é uma conversão direta de dureza Vickers (HV) em LRT conforme disposto na Tabela 2 da norma [24].

Tabela 4. Microdureza Vickers e limite de resistência a tração (LRT) a partir das normas ASTM E140 e ISO 28265-2013.

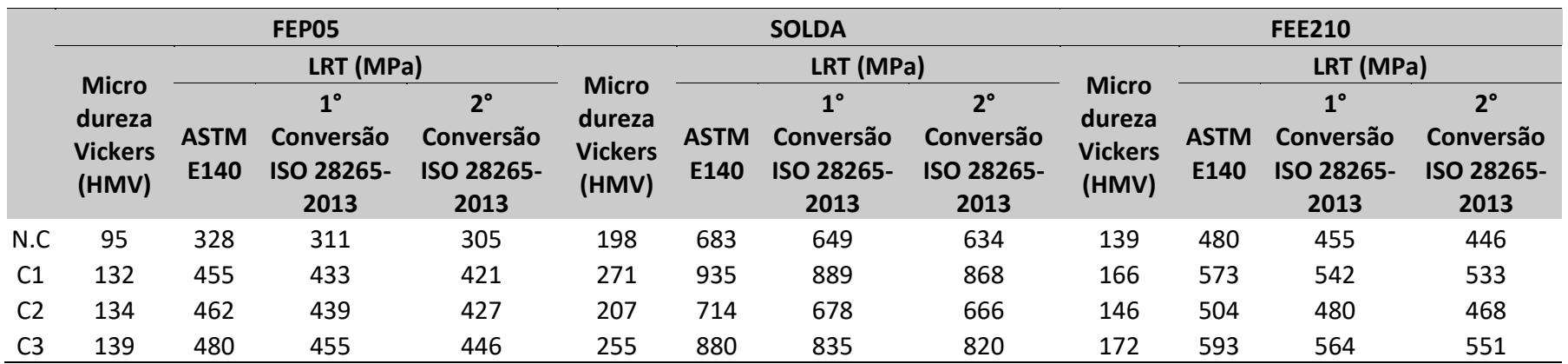

Nota-se que cada uma das conversões utilizadas apresenta um valor distinto. A norma ASTM E140 [23] tem valores superiores a norma ISO 18265-2013 [24]. A diferença entre a ASTM E140 e a $1^{\circ}$ conversão da ISO é menor em relação a $2^{\circ}$ conversão pois utilizam o mesmo método de conversão, isto é, converte-se a dureza Vickers em dureza Brinell e posteriormente obtêm-se o LRT pela Equação 1. 
A diferença entre a $1^{\circ}$ e a $2^{\circ}$ conversão da norma ISO 18265-2013 [24] pode ser atribuída à utilização da Equação 1. Acumulam-se erros devido a duas conversões seguidas, o que aumenta a discrepância entre os valores diretos (tração) e os indiretos (microdureza).

Para realizar a validação dos dados obtidos a partir do ensaio indireto (microdureza), selecionou-se os dados dos ensaios de tração dos materiais de base, da amostra não conformada. Dessa forma foi possível correlacionar com a resistencia mecânica a tração (LRT) obtido através das três conversões (ASTM E140 [23], $1^{\circ}$ e $2^{\circ}$ Conversão ISO 18265-2013 [24]), conforme resultados apresentados na Tabela 5.

Tabela 5. Comparação entre os valores de limite de resistência a tração (LRT) entre o ensaio de tração e os métodos indiretos.

\begin{tabular}{|c|c|c|c|c|c|c|c|c|}
\hline & \multicolumn{4}{|c|}{ FEP05 } & \multicolumn{4}{|c|}{ FEE210 } \\
\hline & $\begin{array}{c}\text { Ensaio de } \\
\text { tração }\end{array}$ & $\begin{array}{c}\text { ASTM } \\
\text { E140 }\end{array}$ & $\begin{array}{c}1^{\circ} \text { Conversão ISO } \\
28265-2013\end{array}$ & $\begin{array}{c}2^{\circ} \text { Conversão ISO } \\
28265-2013 \\
\end{array}$ & $\begin{array}{c}\text { Ensaio de } \\
\text { tração }\end{array}$ & $\begin{array}{c}\text { ASTM } \\
\text { E140 }\end{array}$ & $\begin{array}{c}1^{\circ} \text { Conversão ISO } \\
28265-2013\end{array}$ & $\begin{array}{c}2^{\circ} \text { Conversão ISO } \\
28265-2013\end{array}$ \\
\hline LRT (MPa) & 279 & 328 & 311 & 305 & 344 & 480 & 455 & 446 \\
\hline $\begin{array}{c}\text { Diferença entre o } \\
\text { ensaio direto e } \\
\text { indireto }\end{array}$ & & $18 \%$ & $11 \%$ & $9 \%$ & & $40 \%$ & $32 \%$ & $30 \%$ \\
\hline
\end{tabular}

Observa-se, de modo geral, que os desvios entre as caracterizações mecânicas analisadas são menores para o material FEP05, que possui menor espessura de chapa e menor resistência mecânica em relação ao FEE210. Dessa forma, infere-se que quanto maior for a resistência mecânica do material, mais o método de caracterização indireta se afastará da realidade.

Esse comportamente se deve às normas de conversão de dureza serem atualizadas, em sua última versão, para aços de resistência mecânica relativamente baixa se comparada aos valores dos aços mais recentemente desenvolvidos [23-26]. No caso dos aços IF, como os utilizados no trabalho, considerados de estampagem extra profunda, os valores de dureza tendem a ser mais elevados e, conforme as tabelas, quanto maior a dureza, maior a discrepância.

Os métodos de conversão analisados envolvem transformação de dureza em dureza, e dureza em tensão, dessa forma, apresentam dispersão e erros sistemáticos. A incerteza pode ser afetada por diferentes condições de execução dos testes e mudanças da microestrutura dentro de um mesmo tipo de aço, resultante de diferentes tratamentos térmicos ou trabalho a frio [24].

Dentre os métodos avaliados o que mais se aproximou do ensaio direto foi a $2^{\circ}$ conversão da ISO 18265-2013 [24], 9\% de desvio para o material FEP05 e 30\% para FEE210. Porém também é verificado que quanto maior a dureza, maior é a imprecisão da conversão.

Portanto, para avaliar a região soldada, o melhor método a ser utilizado é a conversão direta disponível na norma ISSO 18265-2013 [24]. Sabe-se que a região soldada possui maior resistência mecânica que os materiais de base, e consequentemente maior dureza. Dessa forma, ressalta-se que a imprecisão da caracterização mecânica dessa região será superior aos $30 \%$ do material de maior resistência mecânica, tornando o método de conversão uma estimativa do comportamento mecânico.

A discrepância encontrada é condizente com o que se espera para este tipo de conversão, conforme apontado em estudos similares, como a resistência mecânica a tração obtida para a linha de solda através da ROM (Rule of mixtures) que tem a descrepancia de 35\% entre o valor calculado (ROM) e o medido (Ensaio de tração) [17]. Para diminuir a divergência de resultados deve-se avaliar e comparar materiais de mesma composição e sob mesmas condições de teste.

Além disso, para verificar de fato a discrepância entre a conversão de dureza em LRT da região soldada, deve-se realizar o ensaio de tração com corpos de prova subsize, conforme apontado em estudos [18,25]. Dessa forma consegue-se avaliar diretamente o comportamento mecânico da solda, tornando o resultado mais próximo ao real.

\section{Conclusões}

O presente trabalhou analisou uma forma de caracterizar mecanicamente a união de chapas fabricadas por Tailor Welded Blanks (TWB), em especial a região soldada. Por meio da realização de ensaios de tração dos materiais de base, ensaios de micrografia, ensaios de microdureza e utilização de métodos disponíveis na literatura e em normas, pode-se concluir que:

$>\quad$ O uso de corpos de prova de tração é útil, para fins qualitativos. O blank se romperá no material de menor espessura, se ocorrer o rompimento na solda, a soldagem apresenta defeitos, e a região soldada não apresenta resistência mecanica a tração maior que os materias de base conforme o esperado;

- Verificou-se leve amaciamento na ZTA no material FEP05, porém não afetou o ensaio de tração qualitativo. Dessa forma, a perda de resistência mecânica do material, no pior dos casos 8,5\%, foi desprezível aos testes realizados; 
- A comparação entre amostra não conformada e conformadas permitiu verificar como a conformação se comportou em cada região analisada, através da deformação do cordão de solda no ensaio de metalografia e elevação da dureza no ensaio de microdureza;

- A caracterização da propriedade mecânica por tração através da dureza ainda não podem ser utilizados para homologação dos TWBs, mas podem ser uteis como métodos adicionais de validação, permitindo inferir o quanto a solda pode contribuir na redução de conformabilidade de TWB. Ainda que haja discrepâncias, consegue-se qualificar de forma objetiva os material e a solda (ZTA E ZF);

- A conversão de dureza Vickers em limite de resistência a tração foi mais eficaz através da norma ISO 28658-2013 pelo método de conversão direto, sem uso de equações da literatura e sem a conversão em outras durezas, diminuindo assim os erros associados as conversões;

- Aponta-se a necessidade de comparação entre o método de conversão e o ensaio de tração com corpo de prova subsize avaliando-se diretamente região soldada.

\section{Agradecimentos}

Os autores agradecem à FIAT Chrysler do Brasil pela contribuição técnica (realização dos ensaios de tração e auxílio técnico para análise de resultados) e o fornecimento dos blanks utilizados para a presente pesquisa.

\section{Referências}

[1] Safdarian R. The effects of strength ratio on the forming limit diagram of tailor-welded blanks. Ironmaking \& Steelmaking. 2016;45(1):17-24.

[2] Chan LC, Chan SM, Cheng CH, Lee TC. Formability and weld zone analysis of Tailor-Welded Blanks for various thickness ratios. Journal of Engineering Materials and Technology. 2005;127(2):179-185. http://dx.doi.org/10.1115/1.1857936.

[3] Gautam V, Raut VM, Kumar DR. Analytical prediction of springback in bending of tailor-welded blanks incorporating effect of anisotropy and weld zone properties. Proceedings of the Institution of Mechanical Engineers, Part L: Journal of Materials: Design and Applications. 2016;232(4):294-306.

[4] Andrade EP, Santos WA, Bracarense AQ. Caracterização mecânica e análise de falhas de chapas fabricadas pelo processo de Tailor Welded Blank submetidas a estampagem profunda. In: Anais do 54 Seminário de Laminação e Conformação; 2017; São Paulo. São Paulo: ABM; 2017. p. 293-301.

[5] Gan Y, Li JX, Chen Y, Li HZ. The optimization method of Tailor Welded Blank forming process based on numerical simulation. Applied Mechanics and Materials. 2014;721:135-139. http://dx.doi.org/10.4028/www.scientific.net/AMM.721.135.

[6] Kinsey BL, Cao J. An analytical model for taior welded blank forming. Journal of Manufacturing Science and Engineering. 2003;125(2):344-354. http://dx.doi.org/10.1115/1.1537261.

[7] Abbasi M, Ketabchi M, Labudde T, Prahl U, Bleck W. New attempt to wrinkling behavior analysis of tailor welded blanks during the deep drawing process. Materials \& Design. 2012;40:407-414. http://dx.doi.org/10.1016/j.matdes.2012.04.015.

[8] Abbasi M, Ketabchi M, Ramazani A, Abbasi M, Prahl U. Investigation into the effects of weld zone and geometric discontinuity on the formability reduction of tailor welded blanks. Computational Materials Science. 2012;59:158-164. http://dx.doi.org/10.1016/j.commatsci.2012.02.039.

[9] Kim J, Ki H. Scaling law for penetration depth in laser welding. Journal of Materials Processing Technology. 2014;214(12):2908-2914. http://dx.doi.org/10.1016/j.jmatprotec.2014.06.025.

[10] Tan CJ, Mori K, Abe Y. Forming of tailor blanks having local thickening for control of wall thickness of stamped products. Journal of Materials Processing Technology. 2008;202(1-3):443-449. http://dx.doi.org/10.1016/j.jmatprotec.2007.10.014.

[11] Min KB, Kim KS, Kang SS. A study on resistance welding in steel sheets using a tailor-welded blank (1st report): evaluation of upset weldability and formability. Journal of Materials Processing Technology. 1998;101(1-3):186-192.

[12] Silva MB, Skjoedt M, Vilaça P, Bay N, Martins PAF. Single point incremental forming of tailored blanks produced by friction stir welding. Journal of Materials Processing Technology. 2009;209(2):811-820. http://dx.doi.org/10.1016/j.jmatprotec.2008.02.057.

[13] Shakeri HR, Buste A, Worswick MJ, Clarke JA, Feng F, Jain M, et al. Study of damage initiation and fracture in aluminum tailor welded blanks made via different welding techniques. Journal of Light Metals. 2002;2(2):95-110. http://dx.doi.org/10.1016/S14715317(02)00028-7.

[14] Assunção E, Quintino L, Miranda R. Comparative study of laser welding in tailor blanks for the automotive industry. International Journal of Advanced Manufacturing Technology. 2009;49(1-4):123-131. 
[15] Chan SM, Chan LC, Lee TC. Tailor-welded blanks of diferent thickness ratios effects on forming limit diagrams. Journal of Materials Processing Technology. 2002;132(1-3):95-101.

[16] Cheng $\mathrm{CH}$, Chan LC, Chow CL. Weldment properties evaluation and formability study of Tailor-Welded Blanks of different thickness combinations and welding orientations. Journal of Materials Science. 2007;42(15):5982-5990. http://dx.doi.org/10.1007/s10853-0061126-0.

[17] Abdullah K, Wild PM, Jeswiet JJ, Ghasempoor A. Tensile testing for weld deformation properties in similar gage tailor welded blanks using the rule of mixtures. Journal of Materials Processing Technology. 2001;112(1):91-97. http://dx.doi.org/10.1016/S09240136(01)00555-6.

[18] Shi MF, Pickett KM, Bhatt KK. Formability issues in the application of tailor welded blank sheets. Warrendale: SAE International; 1993. p. 1-11. (SAE Technical Paper).

[19] Ghoo BY, Keum YT, Kim YS. Evaluation of the mechanical properties of welded metal in tailored steel sheet welded by $\mathrm{CO}_{2}$ laser. Journal of Materials Processing Technology. 2001;113(1-3):692-698. http://dx.doi.org/10.1016/S0924-0136(01)00674-4.

[20] American Society for Testing and Materials. ASTM E8-E8M: standard test methods for tension testing of metallic materials. 16th ed. West Conshohocken: ASTM International; 2016. $30 \mathrm{p}$.

[21] American Society for Testing and Materials. ASTM E3: standard guide for preparation of metallographic specimens. 11th ed. West Conshohocken: ASTM International; 2017. 12 p.

[22] American Society for Testing and Materials. ASTM E384: standard test method for microindentation hardness of materials. 17th ed. West Conshohocken: ASTM International; 2017. 40 p.

[23] American Society for Testing and Materials. E 140-12B: standard hardness conversion tables for metals relationship among brinell hardness, vickers hardness, rockwell hardness, superficial hardness, knoop hardness, scleroscope hardness, and leeb hardness. 12th ed. West Conshohocken: ASTM International; 2013.

[24] International Organization for Standardization. ISO 18265-2013: metallic materials: conversion of hardness values. 2 nd ed. Switzerland: ISO; 2013. 92 p.

[25] Callister WD, Rethwisch DG. Ciência e engenharia de materiais: uma introdução. 9. ed. São Paulo: LTC; 2016. 912 p.

[26] Cetlin PR, Helman H. Fundamentos da conformação: mecânica dos metais. 2. ed. São Paulo: Artliber; 2005. 265 p.

[27] Lee AP, Feltham E, Van Deventer J. Tailor Welded Blank technology for automotive applications. Warrendale: SAE International; 1996. p. 1-14. (SAE Technical Paper, 960817).

[28] Sharma RS, Molian P. Yb: YAG laser welding of TRIP780 steel with dual phase and mild steels for use in tailor welded blanks. Materials \& Design. 2009;30(10):4146-4155. http://dx.doi.org/10.1016/j.matdes.2009.04.033.

[29] Li J, Nayak SS, Biro E, Panda SK, Goodwin F, Zhou Y. Effects of weld line position and geometry on the formability of laser welded high strength low alloy and dual-phase steel blanks. Materials \& Design. 2013;52:757-766. http://dx.doi.org/10.1016/j.matdes.2013.06.021.

[30] Xia M, Sreenivasan N, Lawson S, Zhou Y, Tian Z. A comparative study of formability of diode laser welds in DP980 and HSLA steels. Journal of Engineering Materials and Technology. 2007;129(3):446-452. 